\title{
Oligoclonal Immunoglobulin Gamma Bands and Long-Term Disability Progression in Multiple Sclerosis: A Retrospective Cohort Study
}

Virginija Danylaité Karrenbauer ( $\nabla$ virginija.karrenbauer@ki.se )

Karolinska Institutet Institutionen for klinisk neurovetenskap https://orcid.org/0000-0002-7166-8951

\section{Sahl Bedri}

Karolinska Institute: Karolinska Institutet

Jan Hillert

Karolinska Institutet

Ali Manochehrinia

Karolinska Institutet

Research article

Keywords: Multiple Sclerosis, registry based study, oligoclonal bands, CSF, secondary progression

Posted Date: February 18th, 2021

DOI: https://doi.org/10.21203/rs.3.rs-207684/v1

License: (a) (1) This work is licensed under a Creative Commons Attribution 4.0 International License.

Read Full License 


\section{Abstract \\ Background}

Multiple sclerosis (MS) patients without the typical oligoclonal bands (OCB) distribution of immunoglobulin gamma (IgG) in the cerebrospinal fluid (CSF) has a different genetic background and brain MRI features than OCB-positive MS patients. It is less clear if OCB presence indicates differences in clinical aspects of MS.

\section{Objective}

To determine whether CSF OCB status is associated with long-term disability outcomes.

\section{Methods}

We used Swedish MS register data on clinically definite MS patients with known OCB status. Date of birth, MS onset and date at sustained Expanded Disability Status Scale (EDSS) score milestones 3, 4, 6, date at conversion to secondary progressive (SP) MS, sex, and immunomodulatory treatment (IMTs) duration were collected. Multivariate Cox regression models were used to investigate the association between OCB status and risk of reaching each milestone.

\section{Results}

The OCB-positive group reached disability milestones at an earlier time and at younger age. OCB positivity significantly increased the risk of reaching EDSS score $3.0(\mathrm{HR}=1.29,95 \% \mathrm{Cl}: 1.12$ to $1.48, \mathrm{P}<$ $0.001)$ and $4.0(\mathrm{HR}=1.38,95 \% \mathrm{Cl}: 1.17$ to $1.63, \mathrm{P}<0.001)$ but non-significantly for EDSS 6 . The OCBpositive group had a $20 \%$ higher risk to convert to SPMS.

\section{Conclusion}

Patients with or without CSF OCBs share a risk of long term progression and for conversion to SPMS. Presence of OCBs in the CSF is associated with higher risk to reach EDSS milestones and a higher risk to convert to SPMS. Our findings suggest higher disease modifying effect of OCB presence in the early inflammatory stages of MS.

\section{Background}

Multiple sclerosis (MS) is an immune-mediated demyelinating and neurodegenerative disorder of the central nervous system (CNS).(1) Cerebrospinal fluid (CSF) analysis at the time of diagnosis is used for 
investigation of CSF pathology and for differential diagnosis work up. Routine CSF examination includes measuring the oligoclonal bands (OCB) status, IgG index, albumin ratio and cell counts. OCB in CSF is detected in about $95 \%$ of MS patients. (2) It has been shown that HLA-DRB1*04:04 is associated with OCB-negative MS and carriership of HLA-DRB1*15:01 confers risk for OCB-positive MS.(3-6) We have previously reported that OCB-negative patients MS have less brain white matter atrophy and less regional gray matter loss in specific areas of the brain including basal ganglia, diencephalon, cerebellum and hippocampus.(7) However, findings have been inconsistent with regard to clinical and demographic differences such as age at MS onset, sex ratio and disease severity between the two groups. $(5,8-10)$ Given the paucity of evidence, we aimed at conducting a registry based study to investigate whether OCB carriership confers higher risk of long-term disability worsening in MS.

\section{Methods}

\section{Study population}

We conducted a retrospective registry based study of patients with MS diagnosis according to the McDonald's diagnostic criteria (11-13), registered in the Swedish MS registry. $(14,15)$ Patients with available data on OCB status at the time of MS diagnosis, date of birth, date of MS onset, sex, and duration of exposure to first- and second-line immunomodulatory treatment (IMTs) were included. CSF samples that harbors two or more unique CSF bands in comparison to plasma were considered to be OCB positive.(2) Data was extracted on April 2018.

\section{Study outcomes and definitions}

Study outcomes included risk of reaching sustained Expanded Disability Status Scale (EDSS) score milestones 3.0, 4.0 and 6.0 and conversion to secondary progressive (SP) MS. Sustained EDSS scores were defined as having reached the milestone of interest, having at least one consecutive EDSS measurement after reaching the outcome and not returning back to a lower EDSS score in any consecutive EDSS measurements. Time at conversion to SPMS was defined as the time point when the practicing neurologist had evaluated MS patient having at least one retrospective year of irreversible progress of clinical disability.(16)

\section{Statistical analyses}

Clinical and demographic data at the time of CSF examination were compared between OCB-positive and OCB-negative patients using parametric and non-parametric tests for normally and non-normally distributed data, respectively. The Kaplan-Meier method was used to estimate and compare the median age at reaching EDSS milestones and SP conversion between OCB-positive and OCB-negative groups. Multivariate Cox regression models were used to investigate the influence of OCB status on the risk of reaching each clinical milestone. Models were controlled for sex, age at the onset of MS, calendar year of CSF examination, disease phenotype and exposure to IMTs (as time varying covariate).

\section{Results}


In total, 7322 patients were included of whom 828 patients (11.3\%) were OCB-negative. Age at MS onset was 1.9 years earlier in OCB-positive group compared with OCB-negative $(p<0.001)$. In the OCB-negative group, platform IMTs (interferons, glatiramer acetate, dimethyl fumarate, teriflunomide) were started on average at a 2.5 years higher age in the OCB-negative group compared with OCB-positive $(p<0.001)$. Similarly, more potent IMTs (natalizumab, alemtuzumab, cladribine, fingolimod, rituximab, autologous stem cell transplantation) were started on average at a 3.2 years higher age in the OCB-negative group ( $p$ $<0.001)$. Age at CSF sampling was 2.7 years older in the OCB-negative group $(p<0.001)$. Table 1 summarized characteristics of the study population.

Table 1

Clinicodemographic data of study population, stratified by OCB.

\begin{tabular}{|llll|}
\hline Variables & OCB & OCB & P-value \\
\hline $\mathrm{N}$ & Negative & Positive & \\
\hline Age at onset (mean (SD)) & 828 & 6494 & \\
\hline Age at CSF examination (mean (SD)) & $35.30(10.77)$ & $33.45(10.67)$ & $<0.001$ \\
\hline Male (\%) & $40.79(11.99)$ & $38.13(11.72)$ & $<0.001$ \\
\hline Age at the start of platform IMT (mean (SD)) & $267(32.6)$ & $1894(29.2)$ & 0.049 \\
\hline Age at the start of more potent IMT (mean (SD)) & $40.14(10.47)$ & $37.61(10.76)$ & $<0.001$ \\
\hline Phenotype (\%) & & $39.24(10.92)$ & $<0.001$ \\
\hline PP & $78(10.5)$ & $501(7.9)$ & \\
\hline RR & $460(61.8)$ & $4077(64.1)$ & \\
\hline SP & $206(27.7)$ & $1787(28.1)$ & \\
\hline Reached EDSS 3.0 (\%) & $218(40.0)$ & $2005(44.4)$ & 0.055 \\
\hline Reached EDSS $4.0(\%)$ & $177(27.7)$ & $1511(29.2)$ & 0.466 \\
\hline Reached EDSS 6.0 (\%) & $156(21.7)$ & $1134(19.9)$ & 0.297 \\
\hline Converted to SP (\%) & $171(20.7)$ & $1494(23.0)$ & 0.139 \\
\hline
\end{tabular}

SD: standard deviation; CSF: cerebrospinal fluid; IMT: disease modifying treatment; PP; primary progressive; RR: relapsing remitting; SP: secondary progressive; EDSS: expanded disability status scale.

\section{Age at and risk of sustained EDSS score milestones}

The median age at the sustained EDSS score 3.0 was almost four years younger in OCB-positive patients (57.3 vs. 53.1, Fig. 1A). Similarly, the median ages at the sustained EDSS scores 4.0 and 6.0 were almost 
three (62.5 vs. 60, Fig. 1B) and two (67.8 vs. 66 , Fig. 1C) years younger in OCB-positive compared with OCB-negative, respectively. After controlling for potential confounders, OCB-positive patients had a higher risk of reaching sustained EDSS scores 3.0, 4.0 and 6.0 comparted to OCB-negative patients with HRs of 1.29 (95\%Cl: 1.12 to $1.48, P<0.001, n=5,055), 1.38(95 \% \mathrm{Cl}: 1.17$ to $1.63, P<0.001, n=5,802$ ) and 1.20 (95\%Cl: 0.98 to $1.41, P=0.08, n=6,398)$, respectively.

\section{Age and risk of conversion to SPMS}

After controlling for sex, chronological age, age at the onset of MS, calendar year of CSF examination and exposure to IMTs (as time varying covariate), OCB positivity was associated with increased risk of conversion to SPMS (HR: $1.20,95 \% \mathrm{Cl}: 1.02$ to $1.41, \mathrm{P}=0.03, \mathrm{n}=5,721$ ). Median age of SPMS onset was 58.8 years in OCB-positive group and 61 years in OCB-negative group (Fig. 1D).

\section{Discussion}

In this study, by using the national Swedish MS registry data, that cover $80 \%$ of prevalent MS patients we showed that carriage of CSF OCB is associated with a higher risk to conversion to progressive phase of MS and increase risk of reaching EDSS milestones 3 and 4. We observed clinical and demographic differences between OCB-positive and negative patients in agreement with the previously published genetic studies.(3-7)

In 2013, a meta-analysis regarding OCB role in MS and CIS prevalence and prognosis, including 12,253 MS patients from 71 studies, showed that OCB-positive MS patients had a higher risk to reach disability outcomes with an odds ratio of 1.96.(17) Evidence of intrathecal production of immunoglobulins, mostly $\mathrm{IgG}$, has been an important component of MS diagnostic workout. Although OCB is present in $95 \%$ of MS patients (2) and being highly specific for MS, there was varying importance of CSF OCB role for MS diagnosis during historical evolvement of MS diagnostic criteria. $(11-13,16)$ Revisions of McDonalds criteria at 2017, recommended OCB presence as a criterion to fulfil the dissemination in time in CIS patients with a fulfilled criterion of dissemination in space.(16) Adoption of modern analytical techniques including iso-electric focusing and immunoblotting has greatly increased the proportion of OCB positivity in MS, leaving only around $10 \%$ of the patients consistently being negative in European MS populations. The findings of genetic and imaging differences between the groups (3-7) have prompted a need for renewed assessment of potential clinical differences, as such a distinction would be of importance for how to understand OCB negative MS - as a subgroup of MS or potentially as an entity of its own.

Our study shows that MS subgroups, separated by OCB carriership in CSF, develops similar disability progression patterns in time albeit with varying rates of progression as evident on Kaplan-Meier curves. Hence, it is most likely that these two MS subgroups, defined by OCB carriership in CSF, are very similar and do not create separate disease entities. Our previous publication on familial risk of OCB-positive and OCB-negative groups also indicated that MS lacking OCB is etiologically closely related to the dominant subgroup of OCB positives.(18) 
Disease modifying feature of OCBs maybe most prominent in early stages of MS were inflammatory burden is higher compared with later stages. It is known that inflammatory events like relapses with unfavorable characteristics and number of gadolinium enhancing lesions early in disease are associated with adverse long term outcomes (19) and many studies reported that relapse rate decline with disease duration.(20)

This comprehensive study includes the largest number of OCB negative patients, $828(11.3 \%)$ in total of $7322 \mathrm{MS}$ patients allowing us to calculate a more precise estimation of the prevalence of OCB negative patients in Sweden. Our estimates here is two times the prevalence previously reported by our group previously (5.5\%)(3) and to be considered as representative for Swedish MS population and comparable to OCB-negative MS prevalence reported in Scandinavia (12\%).(5) A study on OCB status and association to specific genetic risk alleles in a Scandinavian cohort (5) also reported a higher prevalence of male gender and older age of onset in OCB negative group.

OCBs are produced by plasma cells which are terminally differentiated B cells. In addition to the presence of OCBs and higher IgG index, the involvement of B cell in MS immunopathology and progression is also supported by the identification of B cell follicles in the meninges of SPMS patients. $(21,22)$ These ectopic $B$ cell follicles can be the site of differentiation of the OCB producing plasma cells.(21) IMTs affect OCB presence in treated MS patients. No evidence of effect of rituximab that primarily target $B$ cells on $\mathrm{OCB}$ presence in CSF has been reported,(23) however, conversion to OCB negative after exposure to natalizumab has been reported in a small subset of patients. $(24,25)$ Histopathological studies had revealed different frequencies of CSF OCB production depending on histopathological MS lesion type: patients with pattern I lesion type harbor OCBs in $88 \%$ of cases and in patients with pattern II and III lesions OCB was present only in $22 \%$. In the latter group two patients were only transiently OCB-positive. (26) Despite that, OCBs are considered to be a diagnostic mainstay feature in MS. $(2,27)$

The presence of OCBs is a clear indication of the inflammatory process in the CNS (28) which is believed even though sometimes debated to be followed by neurodegeneration (29) that is reflected clinically in disability progression. However their specificity and role in the inflammatory process is still under investigation.(28) In a group of CIS and early MS patients, CSF cell counts were observed to be high in OCB positive patients and correlated with percentage of intrathecal IgG production, in addition the latter correlated strongly with percentage of plasma cells in the CSF.(30) Moreover, the levels of serum neurofilament light chain, a well-established marker for neurodegeneration,(31) is higher in OCB positive patients and correlates with CSF IgG levels,(32) indicating higher extension neuro-axonal damage and loss as well as chronic degeneration in OCB positive patients. This is in line with the increased brain atrophy in OCB positive patients but still only confirms the end but not the means or the how.

OCB could be suggested as a potential clinical covariate in randomized clinical trials (RCT). Our current study endpoints were not designed to answer this question, however. In RCT, short term disability progress outcomes are used, such as "confirmed disability progression at 3 months" and "confirmed disability progression" at 6 months. $(33,34)$ International RCT with high number of included RRMS 
patients ( $n>4000$ ) and low EDSS, long time follow up period (3 years) could be characteristics of studies were number of OCB-negative patients would be substantial and time sufficient to evident the OCB effect on disability progression.

Limitation of this study include high number of patient without recorded OCB status. Hence, KaplanMeyer estimates might somewhat be affected by exclusion of old patients that reached milestones but were lacking OCB data. Another limitation of this study is lack of data with regard to factors known to have disease modifying effects such as comorbidities, vitamin D levels, smoking (35-38) or intrinsic laboratory features (presence of IgM bands in $\operatorname{CSF}(39)$ and also clinical characteristics),(40) that could potentially alter final results.

\section{Conclusion}

OCB-positive and OCB-negative MS patients share clinical similarities of long term disability development and conversion to SPMS. However we found that OCB carriership is associated with higher risk of unfavorable outcomes: both reaching disability milestones and SPMS conversion. The effect is small, and more pronounced in early disease stages. Further, our results suggest that OCB status could be considered as a variable in large clinical trials of long term follow up, especially when disability progression is the primary end point.

\section{List Of Abbreviations}

Cl: Confidence interval

CIS: Clinically isolated syndrome

CNS: Central nervous system

CSF: Cerebrospinal fluid

EDSS: Expanded disabilty status scale

HLA: Human leukocyte antigen

HR: Hazard ratio

IgG: Immunoglobulin G

IgM: Immunoglobulin M

IMT: Immunomodulatory treatment

MS: Multiple sclerosis 
OCB: Oligoclonal bands

PP: Primary progressive

RR: Relapsing remitting

SD: Standard deviation

SP: Secondary progressive

SPMS: Secondary Progressive Multiple Sclerosis

\section{Declarations}

Ethical Approval and Consent to participate. Study has been approved by Stockholm's regional ethical committee on 2017-08-16, ethical permission number 2017/1378-31. Patients included in MS registry give their general consent to use anonymized data for future research.

Consent for publication. Not applicable

Availability of supporting data. The data that support the findings of this study are available from Neuroregister.se but restrictions apply to the availability of these data, which were used under license of Neuroregister Research Board and Ethical permission, issued by Stockholm's Regional Ethical Committee for the current study, and so are not publicly available. Data are however available from the authors upon reasonable request and with permission of Swedish Neuroregister Research Board, provided that the requesting person holds ethical permission to analyze the data.

Competing interests. SKB and AM report no conflicts of interest. JH received honoraria for serving on advisory boards for Biogen, Sanofi-Genzyme and Novartis and speaker's fees from Biogen, Merck-Serono, Bayer-Schering, Teva and Sanofi-Genzyme. He has served as P.I. for projects sponsored by, or received unrestricted research support from, Biogen, Merck-Serono, TEVA, Novartis, Sanofi-Genzyme and BayerSchering. VDK has received financial support from Stockholm County Council (grant ALF 20160457); Biogen (recipient of grant and scholarship, PI for project sponsored by); Novartis (Scientific Advisory board member, recipient of scholarship and lecture honoraria); Merc (Scientific Advisory Board member, recipient of lecture honoraria).

Funding. This study was supported by Biogen. Biogen reviewed and provided feedback on the article content to the authors. The authors had full editorial control of article.

Authors' contributions. Conceived and designed the analysis: JH, AM, VDK. Collected the data AM, VDK, $\mathrm{JH}$; Contributed data or analysis tools VDK, JH, AM, SKB. Performed the analysis: AM; Wrote the paper: VDK, AM, SKB, JH. All authors read and approved the final manuscript. 
Acknowledgements. We acknowledge MS patients and Swedish neurologist contributing data to MS registry.

\section{Authors' information. NA}

\section{References}

1. Compston A, Coles A. Multiple sclerosis. Lancet. 2002;359(9313):1221-31.

2. Tumani H, Deisenhammer F, Giovannoni G, Gold R, Hartung HP, Hemmer B, et al. Revised McDonald criteria: the persisting importance of cerebrospinal fluid analysis. Ann Neurol. 2011;70(3):520. author reply 1 .

3. Imrell K, Landtblom AM, Hillert J, Masterman T. Multiple sclerosis with and without CSF bands: clinically indistinguishable but immunogenetically distinct. Neurology. 2006;67(6):1062-4.

4. Kikuchi S, Fukazawa T, Niino M, Yabe I, Miyagishi R, Hamada T, et al. HLA-related subpopulations of MS in Japanese with and without oligoclonal IgG bands. Human leukocyte antigen. Neurology. 2003;60(4):647-51.

5. Mero IL, Gustavsen MW, Saether HS, Flam ST, Berg-Hansen P, Sondergaard HB, et al. Oligoclonal band status in Scandinavian multiple sclerosis patients is associated with specific genetic risk alleles. PLoS One. 2013;8(3):e58352.

6. Leone MA, Barizzone N, Esposito F, Lucenti A, Harbo HF, Goris A, et al. Association of genetic markers with CSF oligoclonal bands in multiple sclerosis patients. PLoS One. 2013;8(6):e64408.

7. Ferreira D, Voevodskaya O, Imrell K, Stawiarz L, Spulber G, Wahlund LO, et al. Multiple sclerosis patients lacking oligoclonal bands in the cerebrospinal fluid have less global and regional brain atrophy. J Neuroimmunol. 2014;274(1-2):149-54.

8. Fukazawa T, Kikuchi S, Sasaki H, Hamada K, Hamada T, Miyasaka K, et al. The significance of oligoclonal bands in multiple sclerosis in Japan: relevance of immunogenetic backgrounds. $\mathrm{J}$ Neurol Sci. 1998;158(2):209-14.

9. Joseph FG, Hirst CL, Pickersgill TP, Ben-Shlomo Y, Robertson NP, Scolding NJ. CSF oligoclonal band status informs prognosis in multiple sclerosis: a case control study of 100 patients. J Neurol Neurosurg Psychiatry. 2009;80(3):292-6.

10. Lechner-Scott J, Spencer B, de Malmanche T, Attia J, Fitzgerald M, Trojano M, et al. The frequency of CSF oligoclonal banding in multiple sclerosis increases with latitude. Mult Scler. 2012;18(7):974-82.

11. Polman CH, Reingold SC, Banwell B, Clanet M, Cohen JA, Filippi M, et al. Diagnostic criteria for multiple sclerosis: 2010 revisions to the McDonald criteria. Ann Neurol. 2011;69(2):292-302.

12. Polman $\mathrm{CH}$, Reingold SC, Edan G, Filippi M, Hartung HP, Kappos L, et al. Diagnostic criteria for multiple sclerosis: 2005 revisions to the "McDonald Criteria". Ann Neurol. 2005;58(6):840-6.

13. McDonald WI, Compston A, Edan G, Goodkin D, Hartung HP, Lublin FD, et al. Recommended diagnostic criteria for multiple sclerosis: guidelines from the International Panel on the diagnosis of 
multiple sclerosis. Ann Neurol. 2001;50(1):121-7.

14. Hillert J, Stawiarz L. The Swedish MS registry - clinical support tool and scientific resource. Acta Neurol Scand. 2015;132(199):11-9.

15. The Swedish MS. registry [Available from: http://www.neuroreg.se/.

16. Thompson AJ, Banwell BL, Barkhof F, Carroll WM, Coetzee T, Comi G, et al. Diagnosis of multiple sclerosis: 2017 revisions of the McDonald criteria. Lancet Neurol. 2018;17(2):162-73.

17. Dobson R, Ramagopalan S, Davis A, Giovannoni G. Cerebrospinal fluid oligoclonal bands in multiple sclerosis and clinically isolated syndromes: a meta-analysis of prevalence, prognosis and effect of latitude. J Neurol Neurosurg Psychiatry. 2013;84(8):909-14.

18. Song J, Karrenbauer V, Manouchehrinia A, Almqvist C, Hillert J, Westerlind H. Similar familial risk in multiple sclerosis subgroups. Mult Scler. 2017;23(13):1782-5.

19. Scott TF. Understanding the impact of relapses in the overall course of MS; refinement of the 2 stage natural history model. J Neuroimmunol. 2017;305:162-6.

20. Confavreux C, Vukusic S. The clinical course of multiple sclerosis. Handb Clin Neurol. 2014;122:34369.

21. Serafini B, Rosicarelli B, Magliozzi R, Stigliano E, Aloisi F. Detection of ectopic B-cell follicles with germinal centers in the meninges of patients with secondary progressive multiple sclerosis. Brain Pathol. 2004;14(2):164-74.

22. Magliozzi R, Howell O, Vora A, Serafini B, Nicholas R, Puopolo M, et al. Meningeal B-cell follicles in secondary progressive multiple sclerosis associate with early onset of disease and severe cortical pathology. Brain. 2007;130(Pt 4):1089-104.

23. Cross AH, Stark JL, Lauber J, Ramsbottom MJ, Lyons JA. Rituximab reduces $B$ cells and T cells in cerebrospinal fluid of multiple sclerosis patients. J Neuroimmunol. 2006;180(1-2):63-70.

24. Harrer A, Tumani H, Niendorf S, Lauda F, Geis C, Weishaupt A, et al. Cerebrospinal fluid parameters of $B$ cell-related activity in patients with active disease during natalizumab therapy. Mult Scler. 2013;19(9):1209-12.

25. Stuve O, Marra CM, Jerome KR, Cook L, Cravens PD, Cepok S, et al. Immune surveillance in multiple sclerosis patients treated with natalizumab. Ann Neurol. 2006;59(5):743-7.

26. Jarius S, Konig FB, Metz I, Ruprecht K, Paul F, Bruck W, et al. Pattern II and pattern III MS are entities distinct from pattern I MS: evidence from cerebrospinal fluid analysis. J Neuroinflammation. 2017;14(1):171.

27. Andersson M, Alvarez-Cermeno J, Bernardi G, Cogato I, Fredman P, Frederiksen J, et al. Cerebrospinal fluid in the diagnosis of multiple sclerosis: a consensus report. J Neurol Neurosurg Psychiatry. 1994;57(8):897-902.

28. Stangel M, Fredrikson S, Meinl E, Petzold A, Stüve O, Tumani H. The utility of cerebrospinal fluid analysis in patients with multiple sclerosis. Nat Rev Neurol. 2013;9(5):267-76. 
29. Milo R, Korczyn AD, Manouchehri N, Stüve O. The temporal and causal relationship between inflammation and neurodegeneration in multiple sclerosis. Multiple Sclerosis Journal. 2019;26(8):876-86.

30. Pfuhl C, Grittner U, Gieß RM, Scheel M, Behrens JR, Rasche L, et al. Intrathecal IgM production is a strong risk factor for early conversion to multiple sclerosis. Neurology. 2019;93(15):e1439.

31. Gaetani L, Blennow K, Calabresi P, Di Filippo M, Parnetti L, Zetterberg H. Neurofilament light chain as a biomarker in neurological disorders. J Neurol Neurosurg Psychiatry. 2019;90(8):870-81.

32. Engel S, Steffen F, Uphaus T, Scholz-Kreisel P, Zipp F, Bittner S, et al. Association of intrathecal pleocytosis and IgG synthesis with axonal damage in early MS. Neurol Neuroimmunol Neuroinflamm. 2020;7(3).

33. Hauser SL, Bar-Or A, Comi G, Giovannoni G, Hartung HP, Hemmer B, et al. Ocrelizumab versus Interferon Beta-1a in Relapsing Multiple Sclerosis. N Engl J Med. 2017;376(3):221-34.

34. Kappos L, Bar-Or A, Cree BAC, Fox RJ, Giovannoni G, Gold R, et al. Siponimod versus placebo in secondary progressive multiple sclerosis (EXPAND): a double-blind, randomised, phase 3 study. Lancet. 2018;391(10127):1263-73.

35. McKay KA, Jahanfar S, Duggan T, Tkachuk S, Tremlett $H$. Factors associated with onset, relapses or progression in multiple sclerosis: A systematic review. Neurotoxicology. 2017;61:189-212.

36. Pittas F, Ponsonby AL, van der Mei IA, Taylor BV, Blizzard L, Groom P, et al. Smoking is associated with progressive disease course and increased progression in clinical disability in a prospective cohort of people with multiple sclerosis. J Neurol. 2009;256(4):577-85.

37. Manouchehrinia A, Hedstrom AK, Alfredsson L, Olsson T, Hillert J, Ramanujam R. Association of PreDisease Body Mass Index With Multiple Sclerosis Prognosis. Front Neurol. 2018;9:232.

38. Healy BC, Ali EN, Guttmann CR, Chitnis T, Glanz BI, Buckle G, et al. Smoking and disease progression in multiple sclerosis. Arch Neurol. 2009;66(7):858-64.

39. Mandrioli J, Sola P, Bedin R, Gambini M, Merelli E. A multifactorial prognostic index in multiple sclerosis. Cerebrospinal fluid IgM oligoclonal bands and clinical features to predict the evolution of the disease. J Neurol. 2008;255(7):1023-31.

40. Vukusic S, Confavreux C. Prognostic factors for progression of disability in the secondary progressive phase of multiple sclerosis. J Neurol Sci. 2003;206(2):135-7.

\section{Figures}



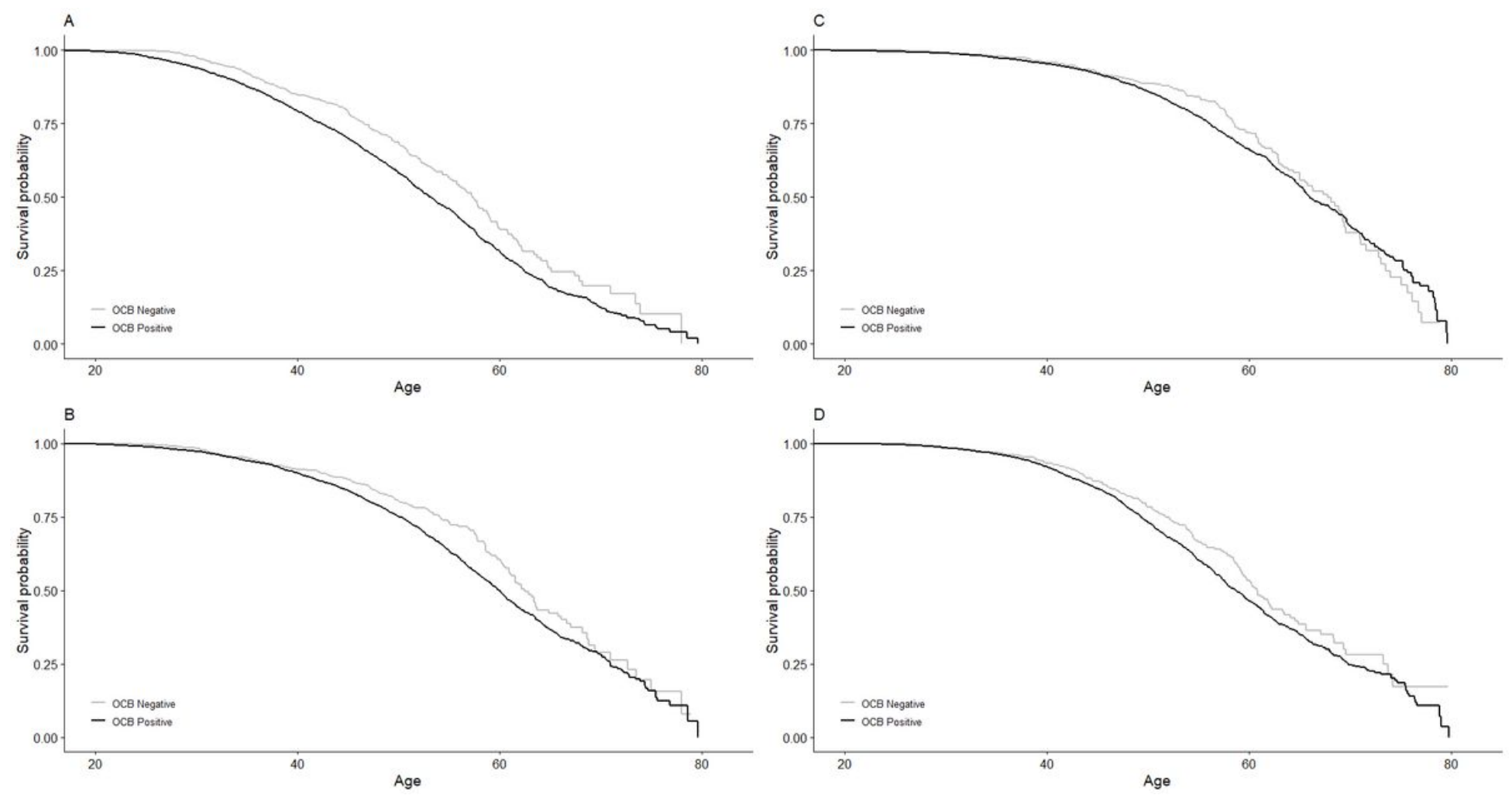

Figure 1

Kaplan-Meier plots (cumulative hazard functions) of reaching EDSS milestones from birth in OCBpositive (black line) and OCB-negative (grey line): 1A EDSS 3, 1B EDSS 4, 1C EDSS 6. 1D: Kaplan-Meier plot of conversion to SP in OCB-positive (black line) and OCB-negative (grey line). 